\title{
INVERSE AND FACTORIZATION OF TRIANGULAR TOEPLITZ MATRICES
}

\author{
ADEM ŞAHIN \\ Received 01 October, 2015

\begin{abstract}
In this paper, we present a new approach for finding the inverse of some triangular Toeplitz matrices using the generalized Fibonacci polynomials and give a factorization of these
\end{abstract} \\ matrices. We also give a new proof of Trudi's formula using our result. \\ 2010 Mathematics Subject Classification: 15A09; 15A23; 11B39 \\ Keywords: triangular Toeplitz matrices, generalized Fibonacci polynomials, matrix factorization
}

\section{INTRODUCTION}

Let $L_{n}$ be the lower triangular Toeplitz matrix:

$$
L_{n}=\left[\begin{array}{cccc}
t_{0} & 0 & \cdots & 0 \\
t_{1} & t_{0} & \ddots & \vdots \\
\vdots & \ddots & \ddots & 0 \\
t_{n-1} & \cdots & t_{1} & t_{0}
\end{array}\right]
$$

where $\left(t_{i}\right)_{i=0,1,2, \ldots, n-1}$ are real and/or complex numbers. In the matrix theory, there is quite an interest in the theory and applications of triangular Toeplitz matrices. There are a number of studies focusing on the linear algebra of the triangular Toeplitz matrices. For example, in [2] the authors discussed the linear algebra of the Pascal matrix, in [8] the authors examined the linear algebra of the $k$-Fibonacci matrix and the symmetric $k$-Fibonacci matrix, in [6] the authors studied the Pell matrix. In [9], Lee et al. defined $n \times n$-Fibonacci matrix and obtained the inverse matrix of the Fibonacci matrix. The Fibonacci matrix $F_{n}=\left[f_{i, j}\right]_{i, j=1,2, \ldots, n}$ and the inverse matrix of $F_{n}$ as follows:

$$
F_{n}=\left[f_{i, j}\right]= \begin{cases}f_{i-j+1}, & \text { for } i-j+1 \geq 0 \\ 0, & \text { for } i-j+1<0\end{cases}
$$


and

$$
F_{n}^{-1}=\left[f_{i, j}^{l}\right]= \begin{cases}1, & \text { for } i=j \\ -1, & \text { for } i-2 \leq j \leq i-1, \\ 0, & \text { otherwise }\end{cases}
$$

where $f_{n}$ is $n$th Fibonacci numbers.

The inverse of Toeplitz matrices was first studied by Trench [18] in 1964 and by Gohberg and Semencul [4] in 1972. In the last decades some papers related to computing the inverse of a nonsingular Toeplitz matrix and the lower triangular Toeplitz matrix were presented, etc. [1,3, 5, 7, 11,16,17, 19,21]. In [16] Merca derived the inverse of triangular Toeplitz matrix using symmetric functions.

In this paper, we obtain the inverse of $\mathrm{n} \times \mathrm{n}$ lower triangular Toeplitz matrix $T_{n}$ as follows:

$$
T_{n}=\left[\begin{array}{cccc}
1 & 0 & \cdots & 0 \\
-t_{1} & 1 & \ddots & \vdots \\
\vdots & \ddots & \ddots & 0 \\
-t_{n-1} & \cdots & -t_{1} & 1
\end{array}\right]
$$

where $\left(t_{i}\right)_{i=1,2, \ldots, n-1}$ are the elements of a ring.

To achieve this goal, we use the generalized Fibonacci polynomials which are general form of a large number of recurrent relation numbers and polynomials. MacHenry $[12,13]$ defined generalized Fibonacci polynomials $\left(F_{k, n}(t)\right)$, where $t_{i}(1 \leq i \leq k)$ are constant coefficients of the core polynomial

$$
P\left(x ; t_{1}, t_{2}, \ldots, t_{k}\right)=x^{k}-t_{1} x^{k-1}-\cdots-t_{k},
$$

which is denoted by the vector $t=\left(t_{1}, t_{2}, \ldots, t_{k}\right) . F_{k, n}(t)$ is defined inductively by

$$
\begin{aligned}
F_{k, n}(t) & =0, n<0 \\
F_{k, 0}(t) & =1, \\
F_{k, n+1}(t) & =t_{1} F_{k, n}(t)+\cdots+t_{k} F_{k, n-k+1}(t) .
\end{aligned}
$$

In addition, in [14] the authors obtained $F_{k, n}(t)(n, k \in \mathbb{N}, n \geq 1)$ as,

$$
F_{k, n}(t)=\sum_{b \vdash n}\left(\begin{array}{c}
|b| \\
b_{1, \ldots,}, b_{n}
\end{array}\right) t_{1}^{b_{1}} \ldots t_{n}^{b_{n}} .
$$

Throughout this paper, the notations $b \vdash n$ and $|b|$ are used instead of $\sum_{j=1}^{n} j b_{j}=n$ and $\sum_{j=1}^{n} b_{j}$, respectively. 
Corollary 1 ([10]). Let $F_{k, n}(t)$ be the generalized Fibonacci polynomials and $H_{-(k, n)}$ be the $n \times n$ lower Hessenberg matrix such that

$$
H_{-(k, n)}=\left[\begin{array}{ccccc}
t_{1} & -1 & 0 & \cdots & 0 \\
t_{2} & t_{1} & -1 & \cdots & 0 \\
\vdots & \vdots & \vdots & \ddots & \vdots \\
t_{k-1} & t_{k-2} & t_{k-3} & \cdots & -1 \\
t_{k} & t_{k-1} & t_{k-2} & \cdots & t_{1}
\end{array}\right] .
$$

Then,

$$
\operatorname{det} H_{-(k, n)}=F_{k, n}(t)
$$

\section{THE INVERSE OF N $\times$ N LOWER TRIANGULAR TOEPLITZ MATRIX}

In this section, we obtain the inverse of matrix $T_{n}(1.1)$. This result was obtained in [16] as a result of the study on symmetry between complete symmetric functions and elementary symmetric functions. We present new approach for this using definition of generalized Fibonacci polynomials.

Theorem 1. Let $T_{n}$ be the $n \times n$ lower triangular Toeplitz matrix in (1.1) and $F_{k, n}(t)$ be the generalized Fibonacci polynomials defined in (1.2), then

$$
\left(T_{n}\right)^{-1}=\left[t_{i, j}\right]= \begin{cases}F_{k, i-j}(t), & \text { for } i-j>0 \\ 1, & \text { for } i-j=0 \\ 0, & \text { otherwise }\end{cases}
$$

Proof.

$$
A_{n}=\left[a_{i, j}\right]= \begin{cases}F_{k, i-j}(t), & \text { for } i-j>0 \\ 1, & \text { for } i-j=0 \\ 0, & \text { otherwise }\end{cases}
$$

and $R_{i}$ is $i$ th row vector of $A_{n}, C_{i}$ is $i$ th column vector of $T_{n}$.

If we show the equation $A_{n} T_{n}=I=\left[e_{i, j}\right]$, the proof is completed. It is obvious that $e_{i j}=0$ for $i<j$ and $e_{i i}=\left\langle R_{i}, C_{i}\right\rangle=1$ from the definitions of $A_{n}$ and $T_{n}$. Now we obtain $e_{i j}$ for $i>j$;

$$
e_{i j}=\left\langle R_{i}, C_{j}\right\rangle=F_{k, i-j+1}(t)-t_{1} F_{k, i-j}(t)-t_{2} F_{k, i-j}(t)-\ldots-t_{k} F_{k, 0}(t) .
$$

From the definition of generalized Fibonacci polynomials and previous equation, we obtain $e_{i j}=0$ for $i>j$, which ends the proof. 
Example 1. We obtain the inverse of matrix $T_{5}$ using Theorem 1;

$$
\begin{gathered}
\left(T_{5}\right)^{-1}=\left[\begin{array}{ccccc}
1 & 0 & 0 & 0 & 0 \\
-t_{1} & 1 & 0 & 0 & 0 \\
-t_{2} & -t_{1} & 1 & 0 & 0 \\
-t_{3} & -t_{2} & -t_{1} & 1 & 0 \\
-t_{4} & -t_{3} & -t_{2} & -t_{1} & 1
\end{array}\right]^{-1} \\
= \\
=\left[\begin{array}{ccccc}
1 & 0 & 0 & 0 \\
F_{k, 1}(t) & 1 & 0 & 0 & 0 \\
F_{k, 2}(t) & F_{k, 1}(t) & 1 & 0 & 0 \\
F_{k, 3}(t) & F_{k, 2}(t) & F_{k, 1}(t) & 1 & 0 \\
F_{k, 4}(t) & F_{k, 3}(t) & F_{k, 2}(t) & F_{k, 1}(t) & 1
\end{array}\right] \\
1 \\
t_{1}
\end{gathered}
$$

Example 2. We obtain " $s_{8,3}$ " for matrix $S_{n}=\left[s_{i, j}\right]_{n \times n}$ which is the inverse of matrix $R_{n}=\left[r_{i j}\right]_{n \times n}=\left\{\begin{array}{cc}1, & i=j, \\ 0, & i<j, \\ j-i, & i>j,\end{array}\right.$ using Theorem 1 and equation (1.3);

$$
s_{8,3}=F_{k, 5}(1,2,3,4,5)=\sum_{b \vdash 5}\left(\begin{array}{c}
|b| \\
b_{1, \ldots,}, b_{5}
\end{array}\right) 1^{b_{1}} \ldots 5^{b_{5}}=55 .
$$

Since $F_{k, n}(t)$ is the general form of the Fibonacci type numbers and polynomials, the results that we obtained are applicable for many polynomials and sequences, such as generalized order- $k$ Fibonacci, Pell and Jacobsthal numbers, generalized bivariate Fibonacci $p$-polynomials, bivariate Jacobsthal $p$-polynomials, Chebyshev polynomial of the second kind and bivariate Pell $p$-polynomials etc.[see Table 1]

The generalized bivariate Fibonacci $p$-polynomials [20] are, for $n>p$,

$$
F_{p, n}(x, y)=x F_{p, n-1}(x, y)+y F_{p, n-p-1}(x, y),
$$

with boundary conditions for $n=1,2, \ldots, p, F_{p, 0}(x, y)=0, F_{p, n}(x, y)=x^{n-1}$. 
Corollary 2. Let $F_{p, n}(x, y)$ be the generalized bivariate Fibonacci p-polynomials defined in (2.1) and $T_{n, p}$ is $n \times n$ lower triangular Toeplitz matrix as

$$
T_{n, p}=\left[c_{i, j}\right]= \begin{cases}1, & \text { for } i-j=0 \\ -x, & \text { for } i-j=1 \\ -y, & \text { for } i-j=p+1 \\ 0, & \text { otherwise }\end{cases}
$$

Then

$$
\left(T_{n, p}\right)^{-1}=\left[a_{i, j}\right]= \begin{cases}F_{p, i-j}(x, y), & \text { for } i-j>0 \\ 1, & \text { for } i-j=0 \\ 0, & \text { otherwise }\end{cases}
$$

Proof. It is obvious that $F_{k-1, n}(x, y)=F_{k, n}(t)$ for $t_{1}=x, t_{i}=0(2 \leq i \leq(k-$ 1)), $t_{k}=y$. So using Theorem 1 , we obtain the required result.

TABLE 1. [20] Cognate polynomial sequences.

\begin{tabular}{|c|c|c||l|}
\hline$x$ & $y$ & $p$ & $F_{p, n}(x, y)$ \\
\hline$x$ & $y$ & 1 & bivariate Fibonacci polynomials $F_{n}(x, y)$ \\
$x$ & 1 & $p$ & Fibonacci p-polynomials $F_{p, n}(x)$ \\
$x$ & 1 & 1 & Fibonacci polynomials $f_{n}(x)$ \\
1 & 1 & $p$ & Fibonacci p-numbers $F_{p}(n)$ \\
1 & 1 & 1 & Fibonacci numbers $F_{n}$ \\
$2 x$ & $y$ & $p$ & bivariate Pell p-polynomials $F_{p, n}(2 x, y)$ \\
$2 x$ & $y$ & 1 & bivariate Pell polynomials $F_{n}(2 x, y)$ \\
$2 x$ & 1 & $p$ & Pell p-polynomials $P_{p, n}(x)$ \\
$2 x$ & 1 & 1 & Pell polynomials $P_{n}(x)$ \\
2 & 1 & 1 & Pell numbers $P_{n}$ \\
$2 x$ & -1 & 1 & second kind Chebysev polynomials $U_{n-1}(x)$ \\
$x$ & $2 y$ & $p$ & bivariate Jacobsthal p-polynomials $F_{p, n}(x, 2 y)$ \\
$x$ & $2 y$ & 1 & bivariate Jacobsthal polynomials $F_{n}(x, 2 y)$ \\
1 & $2 y$ & 1 & Jacobsthal Polynomials $J_{n}(y)$ \\
1 & 2 & 1 & Jacobsthal Numbers $J_{n}$ \\
\hline
\end{tabular}


Corollary 3. Let $U_{n}$ be the $n \times n$ lower triangular Toeplitz matrix as

$$
U_{n}=\left[u_{i, j}\right]= \begin{cases}\left\lfloor\frac{i-j-1}{2}\right\rfloor \\
\sum_{m=0}\left(\begin{array}{c}
i-j \\
2 m+1
\end{array}\right) x^{i-j-2 m}\left(x^{2}-1\right)^{m}, & \text { for } i-j>0, \\
1, & \text { for } i-j=0, \\
0, & \text { otherwise } .\end{cases}
$$

Then

$$
\left(U_{n}\right)^{-1}= \begin{cases}1, & \text { for } i-j=0, i-j=2, \\ -2 x, & \text { for } i-j=1, \\ 0, & \text { otherwise. }\end{cases}
$$

Proof. It is obvious in the Table 1 that $F_{1, n}(x, y)=U_{n-1}(x)$ and

$$
U_{n}(x)=\sum_{m=0}^{\left\lfloor\frac{i-j}{2}\right\rfloor}\left(\begin{array}{c}
i-j+1 \\
2 m+1
\end{array}\right) x^{i-j-2 m}\left(x^{2}-1\right)^{m}
$$

from the definition of the second kind of Chebyshev polynomials. So using Corollary 2 , we obtain the required result.

\section{FACTORIZATIONS OF LOWER TRIANGULAR TOEPLITZ MATRIX}

The set of all square matrices of order $n$ is denoted by $P_{n}$. A matrix $P \in P_{n}$ of the form

$$
P=\left[\begin{array}{cccc}
P_{11} & 0 & \cdots & 0 \\
0 & P_{22} & \ddots & \vdots \\
\vdots & \ddots & \ddots & 0 \\
0 & \cdots & 0 & P_{k k}
\end{array}\right]
$$

in which $P_{i i} \in P_{n_{i}}, i \in\{1,2, \ldots, k\}$, and $\sum_{k=1}^{n} n_{i}=n$, is called block diagonal. Notationally, such a matrix is often indicated as $P=P_{11} \oplus P_{22} \oplus \cdots \oplus P_{k k}$; this is called the direct sum of the matrices $P_{11}, P_{22}, \cdots, P_{k k}$.

In [9], Lee et al. gave the factorization of Fibonacci matrix. Now we consider the factorization of lower triangular Toeplitz matrix. We define the matrices $C_{n}$ and $\widetilde{T_{n}}$ by

$$
C_{n}=\left[\begin{array}{cccc}
1 & 0 & \cdots & 0 \\
-F_{k, 1}(t) & & & \\
\vdots & & I_{n+2} & \\
-F_{k, n+2}(t) & & &
\end{array}\right] \text { and } \widetilde{T_{n}}=[1] \oplus T_{n} .
$$

Theorem 2. Let $T_{n}$ be the $n \times n$ lower triangular Toeplitz matrix in (1.1) and matrices $C_{n}, \widetilde{T_{n}}$ defined in (3.1). Then $\left(\widetilde{T_{k-1}}\right)\left(C_{k-3}\right)=T_{n}$ for $k \geq 3$. 
Proof. For $k=3$, we have $\left(\widetilde{T}_{2}\right)\left(C_{0}\right)=T_{3}$. Letting $k>3$ and applying the definition of generalized Fibonacci polynomials, the proof complete.

Theorem 3. Let $T_{n}$ be the $n \times n$ lower triangular Toeplitz matrix in (1.1), the matrix $C_{n}$ defined in (3.1) and $I_{n}$ be the $n \times n$ identity matrix. Then $T_{n}=\left(I_{n-2} \oplus\right.$ $\left.\left(C_{-1}\right)\right) \ldots\left(I_{1} \oplus\left(C_{n-4}\right)\right)\left(C_{n-3}\right)$.

Proof. The proof is an immediate consequence of Theorem 2.

Example 3. We obtain the factorization of the matrix $T_{5}$ using Theorem 3;

$$
T_{5}=\left(I_{3} \oplus C_{-1}\right)\left(I_{2} \oplus C_{0}\right)\left(I_{1} \oplus C_{1}\right) C_{2},
$$

i.e.

$$
\begin{aligned}
& {\left[\begin{array}{ccccc}
1 & 0 & 0 & 0 & 0 \\
-t_{1} & 1 & 0 & 0 & 0 \\
-t_{2} & -t_{1} & 1 & 0 & 0 \\
-t_{3} & -t_{2} & -t_{1} & 1 & 0 \\
-t_{4} & -t_{3} & -t_{2} & -t_{1} & 1
\end{array}\right]} \\
& =\left[\begin{array}{ccccc}
1 & 0 & 0 & 0 & 0 \\
0 & 1 & 0 & 0 & 0 \\
0 & 0 & 1 & 0 & 0 \\
0 & 0 & 0 & 1 & 0 \\
0 & 0 & 0 & -t_{1} & 1
\end{array}\right]\left[\begin{array}{ccccc}
1 & 0 & 0 & 0 & 0 \\
0 & 1 & 0 & 0 & 0 \\
0 & 0 & 1 & 0 & 0 \\
0 & 0 & -t_{1} & 1 & 0 \\
0 & 0 & -\left(t_{1}^{2}+t_{2}\right) & 0 & 1
\end{array}\right] \\
& {\left[\begin{array}{ccccc}
1 & 0 & 0 & 0 & 0 \\
0 & 1 & 0 & 0 & 0 \\
0 & -t_{1} & 1 & 0 & 0 \\
0 & -\left(t_{1}^{2}+t_{2}\right) & 0 & 1 & 0 \\
0 & -\left(t_{3}+2 t_{1} t_{2}+t_{1}^{3}\right) & 0 & 0 & 1
\end{array}\right]} \\
& {\left[\begin{array}{ccccc}
1 & 0 & 0 & 0 & 0 \\
-t_{1} & 1 & 0 & 0 & 0 \\
-\left(t_{1}^{2}+t_{2}\right) & 0 & 1 & 0 & 0 \\
-\left(t_{3}+2 t_{1} t_{2}+t_{1}^{3}\right) & 0 & 0 & 1 & 0 \\
-\left(t_{4}+2 t_{1} t_{3}+t_{2}^{2}+t_{1}^{4}+3 t_{1}^{2} t_{2}\right) & 0 & 0 & 0 & 1
\end{array}\right] .}
\end{aligned}
$$

Lemma 1. Let $C_{n}$ be the $(n+3) \times(n+3)$ Hessenberg matrix in 3.1 , then

$$
\left(C_{n}\right)^{-1}=\left[\begin{array}{cccc}
1 & 0 & \cdots & 0 \\
F_{k, 1}(t) & & & \\
\vdots & & I_{n+2} & \\
F_{k, n+2}(t) & & &
\end{array}\right]
$$

Proof. The proof is obvious from the matrix product. 
Corollary 4. Let $T_{n}$ be the $n \times n$ lower triangular Toeplitz matrix in (1.1), the matrix $C_{n}$ defined in (3.1) and $I_{n}$ be the $n \times n$ identity matrix. Then $\left(T_{n}\right)^{-1}=$ $\left(C_{n-3}\right)^{-1}\left(I_{1} \oplus\left(C_{n-4}\right)\right)^{-1} \ldots\left(I_{n-2} \oplus\left(C_{-1}\right)\right)^{-1}$.

Proof. The proof follows by a simple calculation using the previous lemma and the equation $\left(I_{k} \oplus\left(C_{n-k-3}\right)\right)^{-1}=I_{k} \oplus\left(C_{n-k-3}\right)^{-1}$.

\section{A NEW PROOF OF TRUDI'S FORMULA}

Merca [15] gave a proof of the Trudi's formula. We give a different proof of this identity using our results.

Theorem 4 (Trudi's formula [15]). Let $m$ be a positive integer. Then

$$
\begin{aligned}
& \operatorname{det}\left[\begin{array}{ccccc}
a_{1} & a_{0} & \cdots & & 0 \\
a_{2} & a_{1} & \cdots & & \\
\vdots & \ddots & \ddots & & \vdots \\
a_{m-1} & a_{m-2} & \cdots & a_{1} & a_{0} \\
a_{m} & a_{m-1} & \cdots & a_{2} & a_{1}
\end{array}\right] \\
= & \sum_{\left(k_{1}, k_{2}, \ldots, k_{m}\right)}\left(\begin{array}{c}
k_{1}+\cdots+k_{m} \\
k_{1}, \cdots, k_{m}
\end{array}\right)\left(-a_{0}\right)^{m-k_{1}-\cdots-k_{m}} a_{1}^{k_{1}} a_{2}^{k_{2}} \cdots a_{m}^{k_{m}}
\end{aligned}
$$

where the summation is over nonnegative integers satisfying $t_{1}+2 t_{2}+\cdots+m t_{m}=$ $m$.

Proof. Using the properties of determinants of Hessenberg matrices, we can write

$$
\begin{aligned}
& \operatorname{det}\left[\begin{array}{cccc}
a_{1} & a_{0} & 0 & 0 \\
a_{2} & a_{1} & \ddots & \vdots \\
\vdots & \ddots & \ddots & a_{0} \\
a_{m} & \cdots & a_{2} & a_{1}
\end{array}\right] \\
& a_{1} \\
& \left.\begin{array}{cccccc}
-a_{0} a_{2} & -1 & 0 & \cdots & 0 \\
\vdots & a_{1} & -1 & \ddots & \vdots \\
(-1)^{m-2} a_{0}^{m-2} a_{m-1} & (-1)^{m-3} a_{0}^{m-3} a_{m-2} & \ddots & & & \\
(-1)^{m-1} a_{0}^{m-1} a_{m} & (-1)^{m-2} a_{0}^{m-2} a_{m-1} & & -1 \\
-a_{0} a_{2} & a_{1}
\end{array}\right]
\end{aligned}
$$

And if we take

$$
t_{1}=a_{1}, t_{2}=-a_{0} a_{2}, \ldots, t_{m}=(-1)^{m-1} a_{0}^{m-1} a_{m}
$$

in equation 1.3 and Corollary 1 , we obtain 


$$
\begin{aligned}
& \operatorname{det}\left[\begin{array}{ccccc}
a_{1} & -1 & 0 & \cdots & 0 \\
-a_{0} a_{2} & a_{1} & -1 & \ddots & \vdots \\
\vdots & \ddots & \ddots & \ddots & 0 \\
(-1)^{m-2} a_{0}^{m-2} a_{m-1} & (-1)^{m-3} a_{0}^{m-3} a_{m-2} & \ddots & a_{1} & -1 \\
(-1)^{m-1} a_{0}^{m-1} a_{m} & (-1)^{m-2} a_{0}^{m-2} a_{m-1} & & -a_{0} a_{2} & a_{1}
\end{array}\right] \\
= & \sum_{\left(k_{1}, k_{2}, \ldots, k_{m}\right)}\left(\begin{array}{c}
k_{1}+\cdots+k_{m} \\
k_{1}, \cdots, k_{m}
\end{array}\right) a_{1}^{k_{1}}\left(-a_{0} a_{2}\right)^{k_{2}} \cdots\left(\left(-a_{0}\right)^{m-1} a_{m}\right)^{k_{m}}
\end{aligned}
$$

Finally, when we make the necessary calculations, equation

$$
\begin{aligned}
& \sum_{\left(k_{1}, k_{2}, \ldots, k_{m}\right)}\left(\begin{array}{c}
k_{1}+\cdots+k_{m} \\
k_{1}, \cdots, k_{m}
\end{array}\right) a_{1}^{k_{1}}\left(-a_{0} a_{2}\right)^{k_{2}} \cdots\left(\left(-a_{0}\right)^{m-1} a_{m}\right)^{k_{m}} \\
= & \sum_{\left(k_{1}, k_{2}, \ldots, k_{m}\right)}\left(\begin{array}{c}
k_{1}+\cdots+k_{m} \\
k_{1}, \cdots, k_{m}
\end{array}\right)\left(-a_{0}\right)^{m-k_{1}-\cdots-k_{m}} a_{1}^{k_{1}} a_{2}^{k_{2}} \cdots a_{m}^{k_{m}}
\end{aligned}
$$

is obtained.

\section{ACKNOWLEDGEMENT}

The authors express their sincere gratitude to the referee for valuable suggestions concerning improvement of the paper.

\section{REFERENCES}

[1] S. Belhaj and M. Dridi, "A note on computing the inverse of a triangular Toeplitz matrix." Appl. Math. Comput., vol. 236, pp. 512-523, 2014.

[2] R. Brawer and M. Pirovino, "The linear algebra of the Pascal matrix." Linear Algebra Appl., vol. 174, pp. 13-23, 1992.

[3] D. Commenges and M. Monsion, "Fast inversion of triangular Toeplitz matrices." IEEE Trans. Automat. Control, vol. AC-29, pp. 250-251, 1984.

[4] I. Gohberg and A. Semencul, "The inversion of finite Toeplitz matrices and their continual analogues." Mat. Issled., vol. 7, pp. 201-223, 1972.

[5] M. Gutknecht and M. Hochbruck, "The stability of inversion formulas for Toeplitz matrices." Linear Algebra Appl., vol. 223-224, pp. 307-324, 1995.

[6] E. K1lic and D. Tasci, "The linear algebra of the Pell matrix." Bol. Soc. Mat. Mexicana, vol. 11, no. 3, pp. 163-174, 2005.

[7] G. Labahn and T. Shalom, "Inversion of Toeplitz matrices with only two standard equations." Linear Algebra Appl., vol. 175, pp. 143-158, 1992.

[8] G. Y. Lee and J. S. Kim, "The linear algebra of the k-Fibonacci matrix." Linear Algebra Appl., vol. 373, pp. 75-87, 2003. 
[9] G. Lee, J. Kim, and S. Lee, "Factorizations and eigenvalues of Fibonacci and symmetric Fibonacci matrices." Fibonacci Quart., vol. 40, no. 3, pp. 203-211, 2002.

[10] H. Li and T. MacHenry, "Permanents and Determinants, Weighted Isobaric Polynomials, and Integer Sequences.” Journal of Integer Sequences, vol. 16, p. 13.3.5., 2013.

[11] F.-R. Lin, W. Ching, and M. Ng, "Fast inversion of triangular Toeplitz matrices." Theor. Comput. Sci., vol. 315, pp. 511-523, 2014.

[12] T. MacHenry, "A Subgroup of the Group of Units in the Ring of Arithmetic Functions." Rocky Mountain J. Math., vol. 29, no. 3, pp. 1055-1065, 1999.

[13] T. MacHenry, "Generalized Fibonacci and Lucas Polynomials and Multiplicative Arithmetic Functions." Fibonacci Quart., vol. 38, pp. 17-24, 2000.

[14] T. MacHenry and K. Wong, "Degree $k$ Linear Recursions $\bmod (p)$ and Number Fields." Rocky Mountain J. Math., vol. 41, no. 4, pp. 1303-1327, 2011.

[15] M. Merca, “A note on the determinant of a Toeplitz-Hessenberg matrix.” Special Matrices, vol. 1, pp. 10-16, 2013, doi: DOI: 10.2478/spma-2013-0003.

[16] M. Merca, "A generalization of the symmetry between complete and elementary symmetric functions," Indian J. Pure Appl. Math., vol. 45, no. 1, pp. 75-89, 2014.

[17] V. Pan, Structured Matrices and Polynomials, Unified Superfast Algorithms. New York: Springer, 2001.

[18] W. F. Trench, "An algorithm for the inversion of finite Toeplitz matrices." J. SIAM, vol. 12, pp. 515-522, 1964.

[19] W. F. Trench, "Inverses of lower triangular Toeplitz matrices.", pp. 1-2, 2009.

[20] N. Tuglu, E. Kocer, and A. Stakhov, "Bivariate fibonacci like p-polynomials." Appl. Math. Comput., vol. 217, pp. 10 239-10 246, 2011, doi: doi:10.1016/j.amc.2011.05.022.

[21] Y. Wei and H. Diao, "On group inverse of singular Toeplitz matrices." Linear Algebra Appl., vol. 399, pp. 109-123, 2005.

Author's address

\section{Adem Şahin}

Gaziosmanpaşa University, Faculty of Education, 60250, Tokat, Turkey

E-mail address: adem.sahinegop.edu.tr, hessenberg.sahin@gmail.com 